\title{
Development and detection of genetically modified crops
}

\author{
Zhao Yu-jia, Fan Pei-lei, Liang Liang, Liu Yin-yin, Zhao Hai-bo and Shen Zheng-shenga \\ Department of Biomedicine, Beijing institute of metrology, Beijing 100029, China
}

\begin{abstract}
Genetically modified crops (GMCs) have been known for the excellent qualities. The commercializing of GMCs has taken great economic and social benefits. However, the bio-security of GMCs was still an issue. To solve this problem, countries around the world were constantly strengthening regulations on planting, processing and detecting of GMCs. This paper reviewed the development of commercialization and detection of GMCs. The difference between protein and nucleic acid detection methods of genetically modified crop was further discussed. This paper will provide new insights for the application of genetically modified crops.
\end{abstract}

\section{Introduction}

Genetically Modified Crops (GMCs) were crops, which genomes have been integrated genes from different organisms by genetic engineering to give them the better characters or qualities ${ }^{[1-3]}$. Since the commercialization of GMCs in 1996, the yield of crops have increased 657.6 million tons that helped more than 65 million farmers to alleviate poverty. Furthermore, GMCs have saved 183 million hectares of land that greatly protected the biological diversity ${ }^{[4]}$. According to the international service for the acquisition of agri-biotech applications (ISAAA), GMCs expected to tackle the food shortages from global population growth ${ }^{[5]}$. GMCs have exhibited important economic, social and ecological value.

\section{The history of GMCs}

Anti-viral tobacco was developed in 1983 and commercialized in 1996. It is the first commercialized GMSs. This marked the commercial era of GMCs has officially arrived ${ }^{[6-8]}$.

Compared with the traditional crops, GMCs exhibit more advantages including insect resistance, herbicide resistance and so on. The commercialization of GMCs has not only brought great economic benefits to farmers, but also provided new solutions to world wiled food shortage problem.

According to ISAAA, GMCs were planted globally at 189.8 million hectares in 67 countries worldwide. From the cultivation area, the United States, Brazil, Argentina, Canada and India are the top five GMCs growth countries, accounting for $91.3 \%$ of global GMCs cultivation in 2017. Large-scale cultivation of GMCs has brought huge economic benefits to the 1.95 billion people in these five countries.

From the cultivation area, the United States, Brazil, Argentina, Canada and India are the top five GMCs growth countries, accounting for $91.3 \%$ of global GMCs cultivation in 2017.

Herbicide-resistant is the main characteristic of GMCs. Herbicide-resistant soybean, rape, corn and cotton have account for $47 \%$ of the total global planting area. Insect and herbicide-resistant is the second main characteristic of GMCs, which account for $41 \%$ of the global planting area. Compound characteristic can reduce the use of both pesticides and herbicides that deeply cut the costs and further protect the environment. Compound characteristic is the future trend of GMCs.

In addition to the traditional four GMCs (corn, soybeans, cotton and rape), genetically modified fruits and vegetables such as beets, pumpkins, potatoes, eggplants, dates, papaya, apples, sugar cane and pineapples have also been commercialized. Furthermore, genetically modified crops with important economic and social benefits, such as rice, wheat, cassava, sweet potato, banana and chickpeas are developing. The cultivated GMCs from countries in 2017 were shown in table 1.

Table 1. Cultivation of GMCs from countries around the world in 2017.

\begin{tabular}{|c|c|c|}
\hline $\mathrm{NO}$. & Country & GMCs \\
\hline 1 & USA & $\begin{array}{c}\text { Corn, Soybean, Cotton, } \\
\text { Rapeseed, Beet, Alfalfa, Papaya, } \\
\text { Pumpkin. Potato, Apple }\end{array}$ \\
\hline 2 & Brazil & Soybean, Corn, Cotton \\
\hline 3 & Argenting & Soybean, Corn, Cotton \\
\hline 4 & Canada & $\begin{array}{c}\text { Rape, Corn, Soybean, Beet, } \\
\text { Alfalfa, Potato }\end{array}$ \\
\hline 5 & India & Cotton \\
\hline 6 & Paraguay & Soybean, Corn, Cotton \\
\hline 7 & Pakistan & Cotton \\
\hline 8 & China & Cotton, Papaya \\
\hline 9 & South Africa & Soybean, Corn, Cotton \\
\hline 10 & Bolivia & Soybean \\
\hline
\end{tabular}

\footnotetext{
$\overline{{ }^{a} \text { Corresponding author: shenzs@bjjl.cn }}$
} 


\section{Biosecurity and Regulatory Policy of GMCs}

\subsection{Biosecurity of GMCs}

Although there are enormous economic and social benefits of GMCs, the debate over the biosecurity of GMCs has never stopped. With the intensified of GMCs commercialization, public concerns about the biosafety of GMCs even grown.

Whether the insertion of genes from plants, animals and microorganisms into a crop is a hazard to people and livestock is the key point of contention for the biosecurity of GMCs. So far, the edible safety experiments for GMCs are mainly takes researched on animals. However, the experiment cycles and generations numbers are limited. Hence, there is no consensus on biosafety of GMCs which is widely accepted by the scientific community and the public. Currently, the biosafety of GMCs was analysed by the international principle of "Substantial equivalence", which was put forward by the International Organization for Economic Cooperation and Development in 1993.

\subsection{Regulatory Policy of GMCs}

GMCs are a double-edged sword, which brings huge economic and social benefits but also has potential biosafety risks. Therefore, countries have set a series of laws to strictly supervise the planting, processing, import and export of GMCs.

EU countries have taken a more cautious attitude to the GMCs, arguing that it is potentially risky of the application of GMCs. So there will be subject to safety evaluation and monitoring. To protect the public's right to know about genetically modified products, the EU stipulates that GMCs must be labelled, and sets the threshold of genetically modified ingredients at $0.9 \%$.

In contrast to the attitude of EU, the United States has taken an active support of GMCs. They believed that genome modified technology was only a process of optimizing the natural evolution to make it controllable and fast. So there is no difference between GMCs and traditional crops. Therefore, the United States was adopted the principle of voluntary labelling of GMCs.

\section{Detection and analysis of GMCs}

To develope rapid and accurate analysis methods is the key point to ensure the supervision of GMCs. Currently, the detection methods of GMCs were mainly divided into two parts, protein detection and nucleic acid detection. To detect the heterogenous proteins of GMCs is a qualitative and quantitative method.

Based on the characteristic that the antibody can specifically bind to the target protein, we can generate detectable signals by coupling the antibody and antigenantibody complex, to realize the qualitative and semiquantitative detection of the heterogenous proteins. The national standard GB/T 19495.8-2004 has seted the detail and applicable scope of protein detection method of GMCs, inclouding the operation process and judgment principle of result credibility of this method.

The protein detection method of GMCs has multiple advantages, such as simplicity, rapidity and low cost. However, proteins are easy to denaturate and degrade, which makes the protein detection method is difficult to detect the processed products of GMCs. In addition, the expression of heterologous proteins in different tissues of plants is also quite different, which is hard for accurate quantification.

The nucleic acid detection of GMCs is a method for qualitative and quantitative analysis of heterologous genes from GMCs (Figure 1). Since the content of DNA in plant cells is relatively stable and it exit in all plant tissues. Therefore, DNA is generally used as a target for detection of GMCs ${ }^{[9-10]}$. Based on the polymerase chain reaction (PCR) of target DNA and analysized by gene sequencing that the insertion site and the sequence of heterologous genes can be detected. So far, the generally used nucleic acid detection PCR including ordinary PCR, nested PCR, semi-nested PCR, multiple PCR, landing PCR, hot-start PCR, PCR-ELISA, competitive quantitative PCR, real-time fluorescent quantitative PCR, digital PCR and so on.

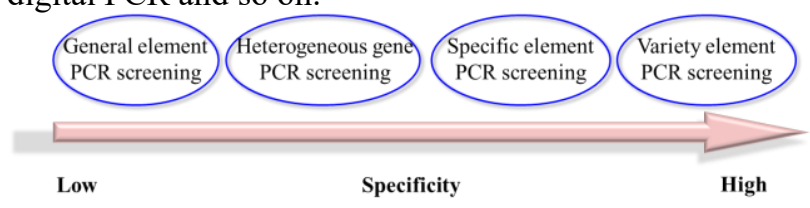

Figure 1. The nucleic acid detection method

Real-time fluorescent quantitative PCR is considered to be an accurate, specific, non-cross contamination and high-throughput quantitative PCR method. Since its introduction in 1992, it has been widely used all over the world. The national standard GB/T 19495.4 2018 "GM products detection in real time fluorescence quantitative polymerase chain reaction (PCR) detection method" and GB/T 19495.5 2018 "GM products detection in real time fluorescence quantitative polymerase chain reaction (PCR) method" have detailed rules on the application scope, operation process and the result credibility principle of this method. It is the authority of the qualitative and quantitative methods for GMCs detection.

The so-called real-time fluorescence quantitative PCR technology means adding fluorescent groups into the PCR reaction system, monitoring the whole PCR process in real time with the accumulation of fluorescence signals, and finally conducting quantitative analysis on the unknown template through standard curve. This method used double labeled fluorescent probes to detect the accumulation of PCR products, which could accurately quantify the content of heterogeneous genes and realized the leap from qualitative to quantitative PCR.

Digital PCR is a new technology which can achieve absolute quantitative. Accoring to the microfluidic technology, nucleic acids will be spread to the chip with multiple micro reactors, make the number of nucleic acid molecule in each reactor is less than or equal to 1 . Then, 
counting was based on the single molecule PCR method. So after PCR cycle, there is a nucleic acid molecular template fluorescent signal presented in the reactor. And there is no fluorescent signal in the reactor with no template. The concentration of nucleic acid in the original solution can be calculated from the relative ratio and the volume of the reactor.The national standard GB/T 33526-2017 'digital PCR detection method for transgenic plant products' has stipulated the applicable scope, operation process and the judgment principle of result credibility of the method. Digital PCR is an internationally recognized authoritative method for the absolute quantification of nucleic acid in GMCs.

Due to the high sensitivity and simple operation procedure of nucleic acid detection, it has developed into a mainstream analysis method for GMCs detection. With the development of GMCs towards to multi-characters and multi-copy numbers, the digital PCR technology that can achieve accurate qualitative and absolute quantification of nucleic acid is expected to become the mainstream of detection methods of GMCs in the future.

\section{Conclusions}

Although the bio-security of GMCs is still a controversial issue, it is undeniable that the better characters of GMCs has brought great economic and social benefits to all countries in the world since the commercialization of GMCs. Tony Blair, the primer ministe of British, has said that "There is no doubt that genetically modified foods are likely to be harmful to human health and the environment. But at the same time this new technology will give more benefits to humanity." Therefore, we believe that with the deepening of human understanding of the origin of life and the rapid development of genetically modified technology, the public will gradually agree on the biosecurity of GMCs.

In this procedure, we should not only speed up the research on GM technology, strengthen the analysis and evaluation of the biosecurity of GMCs, but also follow and improve the regulatory system of GMCs. To bring more benefits for the development of human society.

\section{References}

1. Kamthan A, Chaudhuri A, Kamthan M, et al. Genetically modified (GM) crops: milestones and new advances in crop improvement [J]. Theoretical and Applied Genetics, 2016, 129(9):1639-1655.

2. Chen Hao and Lin Yongjun. Promise and issues of genetically modified crops [J]. Current Opinion in Plant Biology, 2013, 16(2):255-260.

3. Craig W, Tepfer M, Degrassi G, et al. An overview of general features of risk assessments of genetically modified crops [J]. Euphytica, 2008, 164(3):853-880.

4. ISAAA in 2018: Accomplishment Report. http://www.isaaa.org/.

5. South P F, Cavanagh A P, Liu H W, et al. Synthetic glycolate metabolism pathways stimulate crop growth and productivity in the field [J]. Science, 2019, 4: 363(6422).

6. Raman R. The impact of Genetically Modified (GM) crops in modern agriculture: a review [J]. GM Crops and Food, 2017, 8:195-208.

7. Frisvold G and Reeves J. Genetically modified crops: international trade and trade policy effects [J]. International Journal of Food and Agricultural Economics (IJFAEC), 2015, 03.

8. Durham T, Doucet J, Snyder L U. Risk of regulation or regulation of risk? A de minimus framework for genetically modified crops [J]. AgBioForum, 2011, 14(2):61-70.

9. Liu E L, Lu L, Lv Y Z, et al. Event-specific qualitative PCR detection method of genetically modified alfalfa events J101[J]. Journal of Food Safety and Quality, 2015(5):1936-1940.

10. Wang Sen, Li Jian-shuang, Du Xiao-yan. Applications of metabolomics in evaluation of unintended effects of genetically modified crops [J]. Food Science, 2014, 35(9):312-316. 